TRANSACTIONS OF THE

AMERICAN MATHEMATICAL SOCIETY

Volume 357, Number 4, Pages 1545-1564

S 0002-9947(04)03563-9

Article electronically published on September 23, 2004

\title{
A SHARP WEAK TYPE $(p, p)$ INEQUALITY $(p>2)$ FOR MARTINGALE TRANSFORMS AND OTHER SUBORDINATE MARTINGALES
}

\author{
JIYEON SUH
}

\begin{abstract}
If $\left(d_{n}\right)_{n \geq 0}$ is a martingale difference sequence, $\left(\varepsilon_{n}\right)_{n \geq 0}$ a sequence of numbers in $\{1,-1\}$, and $n$ a positive integer, then

$$
P\left(\max _{0 \leq m \leq n}\left|\sum_{k=0}^{m} \varepsilon_{k} d_{k}\right| \geq 1\right) \leq \alpha_{p}\left\|\sum_{k=0}^{n} d_{k}\right\|_{p}^{p} .
$$

Here $\alpha_{p}$ denotes the best constant. If $1 \leq p \leq 2$, then $\alpha_{p}=2 / \Gamma(p+1)$ as was shown by Burkholder. We show here that $\alpha_{p}=p^{p-1} / 2$ for the case $p>2$, and that $p^{p-1} / 2$ is also the best constant in the analogous inequality for two martingales $M$ and $N$ indexed by $[0, \infty)$, right continuous with limits from the left, adapted to the same filtration, and such that $[M, M]_{t}-[N, N]_{t}$ is nonnegative and nondecreasing in $t$. In Section 7 , we prove a similar inequality for harmonic functions.
\end{abstract}

\section{INTRODUCTION}

Most of the paper is devoted to the proof of this sharp inequality for $p>2$ in the simple setting described below. The biconcave function that we construct in Section 3 is used to obtain the upper estimate $p^{p-1} / 2$ for the best constant in this setting. In Section 6 the same function is used to show that $p^{p-1} / 2$ is also an upper estimate of the best constant in the general case. Section 2 contains the proof that $p^{p-1} / 2$ is a lower estimate in the simple case and therefore must also be a lower estimate in the general case. Therefore, $p^{p-1} / 2$ is the best constant in both cases.

Let $\left(f_{n}\right)_{n \geq 0}$ be a sequence, denoted by $f$, of real integrable functions on a probability space $(\Omega, \mathcal{F}, P)$ and $\left(d_{n}\right)_{n \geq 0}$ its difference sequence: $f_{n}=\sum_{k=0}^{n} d_{k}, n \geq 0$. If for all $n \geq 1$, the expectation of the product of $d_{n}$ and $\varphi\left(d_{0}, \cdots, d_{n-1}\right)$ is zero for all real bounded continuous functions $\varphi$ on $\mathbf{R}^{n}$, equivalently, the conditional expectation $E\left(d_{n} \mid d_{0}, \cdots, d_{n-1}\right)=0$ almost everywhere, then $f$ is a martingale. Given such a martingale $f$ and a sequence of numbers $\varepsilon_{n} \in\{1,-1\}$, the transform $g$ of $f$ by $\left(\varepsilon_{n}\right)_{n \geq 0}$ is defined by $g_{n}=\sum_{k=0}^{n} \varepsilon_{k} d_{k}$. Notice that $g$ is also a martingale. The maximal function of $g$ is defined by $g^{*}(\omega)=\sup _{n}\left|g_{n}(\omega)\right|, \omega \in \Omega$, and the $p$-norm of $f$ by $\|f\|_{p}=\sup _{n}\left\|f_{n}\right\|_{p}$.

Here are some typical martingale results specialized to this setting. For martingales $f$ as above (see Doob [16] and the references there to earlier work):

(i) $\|f\|_{1}<\infty \Rightarrow f$ converges a.e.,

Received by the editors February 19, 2003 and, in revised form, November 4, 2003.

2000 Mathematics Subject Classification. Primary 60G44, 60G42; Secondary 60G46.

Key words and phrases. Martingale transform, differential subordination, biconcave majorant.

(C)2004 American Mathematical Society 

(ii) $\lambda^{p} P\left(f^{*} \geq \lambda\right) \leq\|f\|_{p}^{p}$
if $1 \leq p<\infty$ and $\lambda>0$,
(iii) $\left\|f^{*}\right\|_{p} \leq q\|f\|_{p}$
if $1 / p+1 / q=1$ and $1<p<\infty$.

For $f$ and $g$ as above, Burkholder [6] 7] proved that:

(i) $)^{\prime}\|f\|_{1}<\infty \Rightarrow g$ converges a.e.,

(ii) $)^{\prime} \lambda^{p} P\left(g^{*} \geq \lambda\right) \leq \frac{2}{\Gamma(p+1)}\|f\|_{p}^{p} \quad$ if $1 \leq p \leq 2$ and $\lambda>0$,

(iii) $^{\prime}\|g\|_{p} \leq\left(p^{*}-1\right)\|f\|_{p} \quad$ if $1<p<\infty$ and $p^{*}=\max \{p, q\}$,

and the constants in (ii) $)^{\prime}$ and (iii) ${ }^{\prime}$ are the best possible. See Burkholder [8], [9], Bañuelos and Wang [3], and Wang [22] for some of the later related work. Notice that (ii) and (ii)' follow from the special case in which $\lambda$ is replaced by 1 . Also notice that the best constants in (ii) and (ii)' are strikingly different. Inequality (iii) ${ }^{\prime}$ implies that the best constant in (ii) $)^{\prime}$ for $p>2$ is less than or equal to $(p-1)^{p}$. But what is the best constant? In the first part of the paper, we study this natural question. Our answer is contained in the following theorem.

Theorem 1.1. Let $p>2$ and $\lambda>0$. If $f$ is a martingale and $g$ is a transform of $f$ by $\left(\varepsilon_{k}\right)_{k \geq 0}$ as above, then

$$
\lambda^{p} P\left(g^{*} \geq \lambda\right) \leq \frac{p^{p-1}}{2}\|f\|_{p}^{p}
$$

and $p^{p-1} / 2$ is the best constant.

Proof. Let $\beta<p^{p-1} / 2$. In the next section, we shall show that there is a probability space, a martingale $f$ defined on this space, a transform $g$ of $f$ as above, and a positive integer $n$ such that

$$
P\left(\left|g_{n}\right| \geq 1\right)>\beta\left\|f_{n}\right\|_{p}^{p} .
$$

This and the inequality $g^{*} \geq\left|g_{n}\right|$ imply that $p^{p-1} / 2$ is a lower estimate of the best constant.

To prove that $p^{p-1} / 2$ is also an upper estimate, let $f$ be a martingale and $g$ the transform of $f$ by $\left(\varepsilon_{k}\right)_{k>0}$ where $\varepsilon_{k} \in\{1,-1\}$ as above. We can and do assume that $\|f\|_{p}$ is finite. Let $Z_{n}=\left(X_{n}, Y_{n}\right)$ for $n \geq 0$ where

$$
\begin{gathered}
X_{n}=f_{n}+g_{n}=\sum_{k=0}^{n}\left(1+\varepsilon_{k}\right) d_{k}, \\
Y_{n}=f_{n}-g_{n}=\sum_{k=0}^{n}\left(1-\varepsilon_{k}\right) d_{k},
\end{gathered}
$$

so $f_{n}=\frac{X_{n}+Y_{n}}{2}$ and $g_{n}=\frac{X_{n}-Y_{n}}{2}$. Define the function $v$ on $\mathbf{R}^{2}$ by

$$
\begin{aligned}
v(x, y) & =1-\frac{p^{p-1}}{2}\left|\frac{x+y}{2}\right|^{p} \quad \text { if } \quad\left|\frac{x-y}{2}\right| \geq 1, \\
& =-\frac{p^{p-1}}{2}\left|\frac{x+y}{2}\right|^{p} \quad \text { if } \quad\left|\frac{x-y}{2}\right|<1 .
\end{aligned}
$$

Then

$$
P\left(\left|g_{n}\right| \geq 1\right)-\frac{p^{p-1}}{2}\left\|f_{n}\right\|_{p}^{p}=E v\left(Z_{n}\right) .
$$

But $E v\left(Z_{n}\right) \leq 0$ for all $n \geq 0$, as we shall show, so

$$
P\left(\left|g_{n}\right| \geq 1\right) \leq \frac{p^{p-1}}{2}\left\|f_{n}\right\|_{p}^{p}
$$


and (1.1) easily follows by a standard stopping-time argument, which here goes as follows. Let $\tau(\omega)=\inf \left\{n \geq 0:\left|g_{n}(\omega)\right| \geq 1\right\}$ so that $P\left(\max _{0 \leq k \leq n}\left|g_{k}\right| \geq 1\right)=$ $P\left(\left|g_{\tau \wedge n}\right| \geq 1\right)$, apply (1.2) to the martingale $\left(f_{\tau \wedge n}\right)_{n \geq 0}$ and its transform $\left(g_{\tau \wedge n}\right)_{n \geq 0}$ and then use the inequality $\left\|f_{\tau \wedge n}\right\|_{p} \leq\left\|f_{n}\right\|_{p}$. This yields $P\left(g^{*}>1\right) \leq p^{p-1} / 2\|f\|_{p}$ for all $f$ and $g$ as above and gives (1.1) with strict inequality on the left side. The limit of $\eta P\left(g^{*}>\eta\right)$ as $\eta \uparrow \lambda$ is $\lambda P\left(g^{*} \geq \lambda\right)$ so (1.1) holds as stated. In Section 3, we show that there exists a biconcave majorant $u$ of $v$ on $\mathbf{R}^{2}$ satisfying $u(0,0)=0$. The function $u$ has the further property that $u(0, y)=u(0,-y)$ and $u(x, 0)=u(-x, 0)$. Therefore, $E v\left(Z_{n}\right) \leq E u\left(Z_{n}\right)$ and $u(x, y) \leq 0$ for all $x, y \in \mathbf{R}$ satisfying $x y=0$. The next step is to show that $E u\left(Z_{n}\right) \leq E u\left(Z_{n-1}\right)$ for all $n \geq 1$. To do this assume that $\varepsilon_{n}=1$, the case $\varepsilon_{n}=-1$ being similar. Then by the conditional form of the Jensen inequality for concave functions, we have that almost everywhere

$$
\begin{aligned}
E\left[u\left(Z_{n}\right) \mid d_{0}, \cdots, d_{n-1}\right] & =E\left[u\left(X_{n-1}+2 d_{n}, Y_{n-1}\right) \mid d_{0}, \cdots, d_{n-1}\right] \\
& \leq u\left(X_{n-1}+2 E\left(d_{n} \mid d_{0}, \cdots, d_{n-1}\right), Y_{n-1}\right) \\
& =u\left(Z_{n-1}\right) .
\end{aligned}
$$

Taking expectations of both sides gives $E u\left(Z_{n}\right) \leq E u\left(Z_{n-1}\right)$. So

$$
E u\left(Z_{n}\right) \leq \cdots \leq E u\left(Z_{0}\right) .
$$

But $E u\left(Z_{0}\right) \leq 0$ since $u\left(Z_{0}\right)=u\left(\left(1+\varepsilon_{0}\right) d_{0},\left(1-\varepsilon_{0}\right) d_{0}\right) \leq 0$, in which the product of $\left(1+\varepsilon_{0}\right) d_{0}$ and $\left(1-\varepsilon_{0}\right) d_{0}$ is zero. Therefore, $E v\left(Z_{n}\right) \leq E u\left(Z_{n}\right) \leq 0$.

The function $u$ that we have used in this proof is concave along horizontal and vertical lines; it is also concave along every line of positive slope as we prove in Section 4. We use this property in Section 5 to generalize Theorem 1.1 to differentially subordinate martingales. A sharp weak type inequality for martingale transforms more general than the plus-and-minus-one transforms of Theorem 1.1 follows at once, and by approximation, so does a similar inequality for stochastic integrals. The results of Section 4 and 5 lead to the proof in Section 6 of the following theorem, the main result of this paper. In this theorem the probability space $(\Omega, \mathcal{F}, P)$ is complete, the filtration $\left(\mathcal{F}_{t}\right)_{t \geq 0}$ is right-continuous, and $\mathcal{F}_{0}$ contains all the sets of measure 0 .

Theorem 1.2. Let $p>2$ and $\lambda>0$. If $M$ and $N$ are right-continuous martingales with limits from the left, adapted to the filtration $\left(\mathcal{F}_{t}\right)_{t \geq 0}$, and $[M, M]_{t}-[N, N]_{t}$ is nonnegative and nondecreasing in $t$ for $t \in[0, \infty)$, then

$$
\lambda^{p} P\left(N^{*} \geq \lambda\right) \leq \frac{p^{p-1}}{2}\|M\|_{p}^{p}
$$

and the constant $p^{p-1} / 2$ is the best possible.

For background on the quadratic-variation process $[M, M]$, see Dellacherie and Meyer [15] or Protter [20]. The condition that $[M, M]_{t}-[N, N]_{t}$ is nonnegative and nondecreasing in $t$ for $t \geq 0$ was introduced and used by Bañuelos and Wang [3] and Wang [22]; see also [4], [5].

In the differential subordination setting of Section 5, the Bañuelos-Wang condition takes the form

$$
\sum_{k=0}^{n}\left(d_{k}^{2}-e_{k}^{2}\right)
$$


is nonnegative and nondecreasing in $n$ and is equivalent to the condition (5.1). In the stochastic integral setting of $\left[9\right.$ where the $L^{p}$-norm of $\int_{0}^{t} U_{s} d M_{s}$ and $\int_{0}^{t} V_{s} d M_{s}$ are compared under the condition that the predictable processes $U$ and $V$ satisfy

$$
\left|V_{s}(\omega)\right| \leq\left|U_{s}(\omega)\right| \text { for all } \omega \in \Omega \text { and } s \geq 0,
$$

it takes the form

$$
\int_{0}^{t}\left(\left|U_{s}\right|^{2}-\left|V_{s}\right|^{2}\right) d[M, M]_{s}
$$

is nonnegative and nondecreasing in $t$. The Bañuelos-Wang condition makes it possible to obtain sharp inequalities for a larger class of martingale pairs $(M, N)$. In this setting the special functions needed for the stochastic integral and discrete-time cases again come into play. It is not known for most sharp martingale inequalities, however, if the less restrictive condition (i) $[N, N]_{t} \leq[M, M]_{t}$ for all $t \geq 0$, or the even less restrictive condition (ii) $[N, N]_{\infty} \leq[M, M]_{\infty}$ would suffice; see Section 6 of 9 .

In Section 7, we use the inequality (1.3) to prove an analogous inequality for harmonic functions.

\section{A Lower estimate of the Best constant}

Let $p>2$ and $0<\beta<p^{p-1} / 2$. Then choose $\theta \in\left(0, \frac{p-1}{p}\right)$ so that

$$
\beta<(1-\theta)^{p} p^{p-1} / 2 \text {. }
$$

Finally, choose an odd positive integer $M$ satisfying both $M>p$ and

$$
(M-1)^{2}>\frac{4}{\theta^{p}}\left(\frac{p-1}{p}\right)^{p-1} p(p-1)(p-2)\left(\theta^{p-3}+\theta^{3-p}\right) .
$$

In this section, we construct a martingale $F$ such that $G$, the transform of $F$ by the sequence $(-1,1,-1,1, \cdots)$, satisfies

$$
P\left(\left|G_{M}\right| \geq 1\right)>\beta\|F\|_{p}^{p},
$$

showing that the best constant for the inequality (1.1) must be greater than or equal to $p^{p-1} / 2$. For convenience in the proof, we define $\delta$ by $(M-1) \delta=\frac{p-1}{p}-\theta$.

Consider the following $\mathbf{R}^{2}$-valued martingale $Z$, one that is also Markov. Here $Z_{n}=\left(X_{n}, Y_{n}\right)$, a function with all of its values in the set

$$
\left\{c_{1}, \cdots, c_{M}, c_{*}, z_{0}, \cdots, z_{M-1}\right\} \subset \mathbf{R}^{2}
$$

where the elements of this set are defined as follows: $z_{0}=(\theta,-\theta)$; if $n$ is an odd integer such that $1 \leq n \leq M-2$, then

$$
z_{n}=(\theta+n \delta,-\theta-(n-1) \delta) ;
$$

if $n$ is an even integer such that $2 \leq n \leq M-1$, then

$$
z_{n}=(\theta+(n-1) \delta,-\theta-n \delta) ;
$$

if $n$ is an odd integer such that $1 \leq n \leq M$, then

$$
c_{n}=\left(\frac{p-3}{p-1}(\theta+(n-1) \delta),-\theta-(n-1) \delta\right) ;
$$

if $n$ is an even integer such that $2 \leq n \leq M-1$, then

$$
c_{n}=\left(\theta+(n-1) \delta,-\frac{p-3}{p-1}(\theta+(n-1) \delta)\right) ;
$$


and

$$
c_{*}=\left(\frac{p+1}{p},-\frac{p-1}{p}\right) .
$$

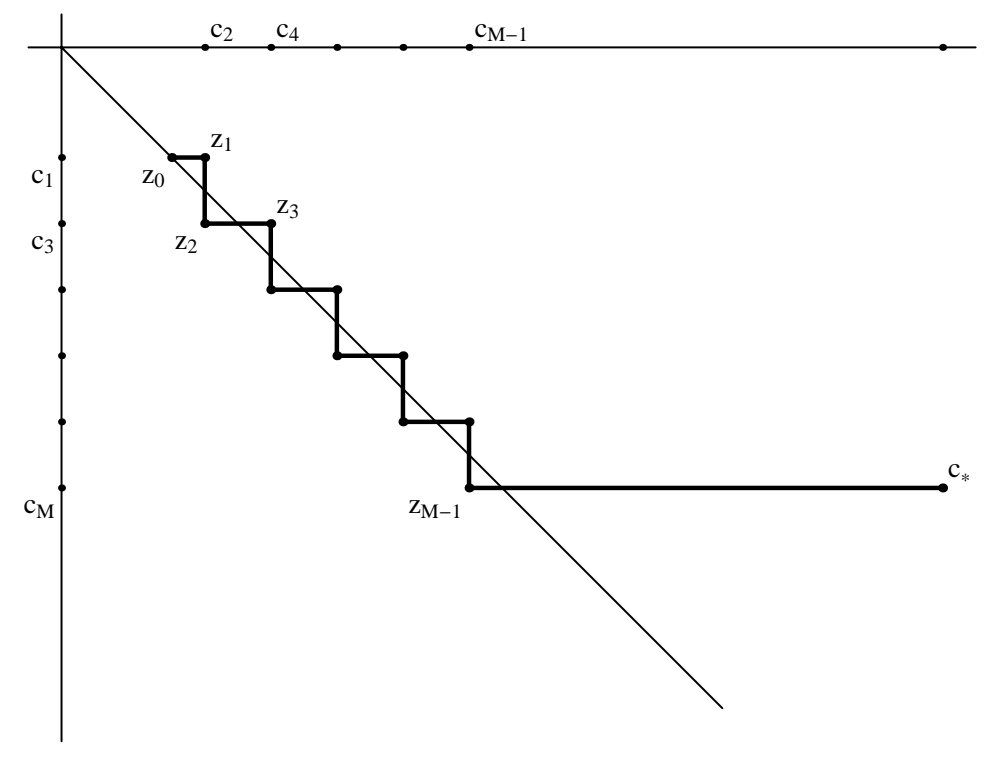

Figure 1 . One of the paths of $Z$ for the case $p=3$

Before describing the probability framework, we remark that intuitively $Z_{n}$ represents a Markov particle's location at time $n ; Z$ starts at $z_{0}$ and then moves to $z_{1}$ or $c_{1}$; if not $c_{1}$, then it moves successively through $z_{1}, z_{2}, \cdots, z_{n-1}$ and then to $z_{n}$ or $c_{n}$ if $1 \leq n<M-1$; once it arrives at a $c_{n}$ it stops; if it ever arrives at $z_{M-1}$, then it moves to $c_{M}$ or to $c_{*}$ and then stops. Figure 1 illustrates one of the paths of $Z$ in the case $p=3$.

Let $\Omega$ denote the set $\left\{c_{1}, c_{2}, \cdots, c_{M}, c_{*}\right\}, \mathcal{F}$ the $\sigma$-algebra of all subsets of $\Omega$, and $P$ the probability measure that assigns weight $\pi_{n}$ to $c_{n}$ if $1 \leq n \leq M$ and weight $\pi_{*}$ to $c_{*}$. The weights depend on $p, \theta$, and $M$ as does $\Omega$. These weights need not be given explicitly here because the probability structure will be described below in a more useful way.

We define $Z$ on $\Omega$ as follows: $Z_{0}(\omega)=z_{0}$ for all $\omega \in \Omega$; if $1 \leq m \leq n \leq M$, then $Z_{n}\left(c_{m}\right)=c_{m}$; if $1 \leq n \leq M-1$, then $Z_{n}(\omega)=z_{n}$ for all $\omega \in\left\{c_{n+1}, \cdots, c_{M}, c_{*}\right\}$; $Z_{M}\left(c_{*}\right)=c_{*} ;$ if $n>M$, then $Z_{n}=Z_{M}$.

It is clear that $Z$ is Markov with $P\left(Z_{0}=z_{0}\right)=1$. To force $Z$ to be a martingale, we assume that (here $\left(a_{n}, b_{n}\right)=c_{n},\left(a_{*}, b_{*}\right)=c_{*}$, and $\left.\left(x_{n}, y_{n}\right)=z_{n}\right)$

$$
\begin{aligned}
& P\left(Z_{1}=z_{1}\right)=\frac{x_{0}-a_{1}}{x_{1}-a_{1}} \\
& P\left(Z_{1}=c_{1}\right)=\frac{\delta}{x_{1}-a_{1}}
\end{aligned}
$$


if $n$ is an even integer such that $2 \leq n \leq M-1$, then

$$
\begin{aligned}
& P\left(Z_{n}=z_{n} \mid Z_{n-1}=z_{n-1}\right)=\frac{b_{n}-y_{n-1}}{b_{n}-y_{n}}, \\
& P\left(Z_{n}=c_{n} \mid Z_{n-1}=z_{n-1}\right)=\frac{2 \delta}{b_{n}-y_{n}}
\end{aligned}
$$

if $n$ is an odd integer such that $3 \leq n \leq M-2$, then

and

$$
\begin{aligned}
& P\left(Z_{n}=z_{n} \mid Z_{n-1}=z_{n-1}\right)=\frac{x_{n-1}-a_{n}}{x_{n}-a_{n}}, \\
& P\left(Z_{n}=c_{n} \mid Z_{n-1}=z_{n-1}\right)=\frac{2 \delta}{x_{n}-a_{n}}
\end{aligned}
$$

$$
\begin{aligned}
P\left(Z_{M}=c_{*} \mid Z_{M-1}=z_{M-1}\right) & =\frac{x_{M-1}-a_{M}}{a_{*}-a_{M}}, \\
P\left(Z_{M}=c_{M} \mid Z_{M-1}=z_{M-1}\right) & =\frac{a_{*}-x_{M-1}}{a_{*}-a_{M}} .
\end{aligned}
$$

Observe that $E\left[Z_{n} \mid Z_{n-1}\right]=Z_{n-1}$ so by the Markov property

$$
E\left[Z_{n} \mid Z_{0}, \ldots, Z_{n-1}\right]=Z_{n-1} \text { if } n \geq 1 .
$$

Therefore, $Z$ is a martingale. Also, $Z_{0}=\left(X_{0}, Y_{0}\right)=(\theta,-\theta)$ and if $n \geq 1$, then

$$
X_{n}=\theta+\sum_{k=1}^{n}\left(1+\varepsilon_{k}\right) d_{k} \quad \text { and } \quad Y_{n}=-\theta+\sum_{k=1}^{n}\left(1-\varepsilon_{k}\right) d_{k},
$$

where $\varepsilon_{k}=(-1)^{k+1}$ and $d_{n}$ is given by $X_{n}-X_{n-1}=\left(1+\varepsilon_{n}\right) d_{n}$ if $n$ is odd and by $Y_{n}-Y_{n-1}=\left(1-\varepsilon_{n}\right) d_{n}$ if $n$ is even. Consequently, $f$ and $g$ defined by $f_{0}=g_{0}=0$ and, for $n \geq 1$, by

$$
f_{n}=\sum_{k=1}^{n} d_{k} \quad \text { and } \quad g_{n}=\sum_{k=1}^{n} \varepsilon_{k} d_{k},
$$

are also martingales with $g$ being a transform of $f$ by $\left(\varepsilon_{k}\right)_{k \geq 0}$. For $n \geq 0$,

$$
f_{n}=\frac{X_{n}+Y_{n}}{2} \text { and } \theta+g_{n}=\frac{X_{n}-Y_{n}}{2} .
$$

Using the function $v$ defined in (1.2), we have that

$$
\begin{aligned}
E\left[v\left(Z_{M}\right)\right] & =P\left(\left|\frac{X_{M}-Y_{M}}{2}\right| \geq 1\right)-\frac{p^{p-1}}{2} E\left[\left|\frac{X_{M}+Y_{M}}{2}\right|^{p}\right] \\
& =P\left(\left|\theta+g_{M}\right| \geq 1\right)-\frac{p^{p-1}}{2}\|f\|_{p}^{p} .
\end{aligned}
$$

Here $\left\|f_{M}\right\|_{p}=\|f\|_{p}$ because $\left\|f_{0}\right\|_{p} \leq \cdots \leq\left\|f_{M}\right\|_{p}=\left\|f_{M+1}\right\|_{p}=\cdots$. The main step in the proof that $p^{p-1} / 2$ is a lower estimate for the best constant is to show that

$$
E\left[v\left(Z_{M}\right)\right]>0 .
$$

If (2.4) holds, then by (2.3),

$$
P\left(\left|g_{M}\right| \geq 1-\theta\right) \geq P\left(\left|\theta+g_{M}\right| \geq 1\right)>\frac{p^{p-1}}{2}\|f\|_{p}^{p} .
$$


Using this and (2.1), we obtain

$$
P\left(\left|G_{M}\right| \geq 1\right)>(1-\theta)^{p} \frac{p^{p-1}}{2}\|F\|_{p}^{p}>\beta\|F\|_{p}^{p}
$$

where $F$ is the martingale $(1-\theta)^{-1} f$, and $G=(1-\theta)^{-1} g$, the transform of $F$ by $(-1,1,-1,1, \cdots)$. So provided (2.4) holds, $p^{p-1} / 2$ is a lower estimate of the best constant.

To prove (2.4), define $\phi: \mathbf{R} \times\left[-\frac{p-1}{p}, 0\right) \rightarrow \mathbf{R}$ and $\psi:\left(0, \frac{p-1}{p}\right] \times \mathbf{R} \rightarrow \mathbf{R}$ by

$$
\begin{array}{r}
\phi(x, y)=[(p-2) y+p x](-y)^{p-1}, \\
\psi(x, y)=[(2-p) x-p y] x^{p-1},
\end{array}
$$

and notice, for example, that $\phi_{x x}(x, y)=0$ but that

$$
\begin{gathered}
\psi_{x x}(x, y)=-p(p-1)(p-2)(x+y) x^{p-3}, \\
\psi_{x x x}(x, y)=-p(p-1)(p-2)[-y+(x+y)(p-2)] x^{p-4} .
\end{gathered}
$$

These imply that $\psi_{x x}(-y, y)=0$ and $\psi_{x x x}(x, y)<0$ if $x \geq-y>0$. If $-\frac{p-1}{p}<y<$ 0 and $\frac{3-p}{p-1} y \leq x \leq-y$, let

$$
u(x, y)=\frac{1}{4}\left(\frac{p}{p-1}\right)^{p-1} \phi(x, y)
$$

and also if $y=-\frac{p-1}{p}$ and $\frac{p-3}{p} \leq x \leq \frac{p+1}{p}$. If $0<x \leq \frac{p-1}{p}$ and $-x<y \leq \frac{3-p}{p-1} x$, let

$$
u(x, y)=\frac{1}{4}\left(\frac{p}{p-1}\right)^{p-1} \psi(x, y) .
$$

By the definitions of $\phi$ and $\psi$, the function $u$ is continuous, and has continuous first and second partial derivatives on the interior of its domain of definition.

Observe that $u(\omega)=v(\omega)$ for all $\omega \in \Omega$ : both $u\left(c_{*}\right)$ and $v\left(c_{*}\right)$ are equal to $(2 p-1) /(2 p)$ and if $1 \leq n \leq M$, then both $u\left(c_{n}\right)$ and $v\left(c_{n}\right)$ are equal to

$$
\frac{-p^{p-1}}{2(p-1)^{p}}(\theta+(n-1) \delta)^{p} .
$$

Therefore, $E\left[v\left(Z_{M}\right)\right]=E\left[u\left(Z_{M}\right)\right]$ and the problem is reduced to showing that

$$
E\left[u\left(Z_{M}\right)\right]>0 .
$$

The calculation of $E\left[u\left(Z_{0}\right)\right]$ shows that

$$
E\left[u\left(Z_{M}\right)\right]=\frac{\theta^{p}}{2}\left(\frac{p}{p-1}\right)^{p-1}+\sum_{n=1}^{M} E\left[u\left(Z_{n}\right)-u\left(Z_{n-1}\right)\right] .
$$

So (2.8) will follow if we can prove that

$$
\sum_{n=1}^{M} E\left[u\left(Z_{n}\right)-u\left(Z_{n-1}\right)\right]>-\frac{\theta^{p}}{4}\left(\frac{p}{p-1}\right)^{p-1} .
$$


By Taylor's formula with Lagrange's remainder,

$$
\begin{aligned}
E\left[u\left(Z_{1}\right)-u\left(Z_{0}\right)\right]= & {\left[u\left(z_{0}+(\delta, 0)\right)-u\left(z_{0}\right)\right] \frac{x_{0}-a_{1}}{x_{1}-a_{1}} } \\
& +\left[u\left(c_{1}\right)-u\left(z_{0}\right)\right] \frac{\delta}{x_{1}-a_{1}} \\
= & {\left[u_{x}\left(z_{0}\right) \delta+\frac{\delta^{2}}{2} R_{1}\right] \frac{x_{0}-a_{1}}{x_{1}-a_{1}} } \\
& +\left[u_{x}\left(z_{0}\right)\left(a_{1}-x_{0}\right)\right] \frac{\delta}{x_{1}-a_{1}} \\
= & \frac{\delta^{2}}{2} R_{1} \frac{x_{0}-a_{1}}{x_{1}-a_{1}}
\end{aligned}
$$

where, for some $\delta^{*} \in(0, \delta), R_{1}=u_{x x}\left(z_{0}+\left(\delta^{*}, 0\right)\right)$. By (2.6) and (2.7),

$$
0>R_{1}>u_{x x}\left(z_{0}+(\delta, 0)\right)=-p(p-1)(p-2) \delta x_{1}^{p-3} .
$$

So (2.10),$\left(x_{0}-a_{1}\right) /\left(x_{1}-a_{1}\right) \in(0,1)$, and $\theta<x_{1} \leq \frac{p-1}{p}<1<\frac{1}{\theta}$ imply that

$$
E\left[u\left(Z_{1}\right)-u\left(Z_{0}\right)\right]>-\frac{1}{2} p(p-1)(p-2) \delta^{3} x_{1}^{p-3}>-C_{p, \theta} \delta^{3}
$$

where $C_{p, \theta}=p(p-1)(p-2)\left(\theta^{3-p}+\theta^{p-3}\right)$. If $2 \leq n \leq M$, then

$$
E\left[u\left(Z_{n}\right)-u\left(Z_{n-1}\right)\right]>-C_{p, \theta} \delta^{3}
$$

also holds as we shall see. Since $Z_{n}(\omega) \neq Z_{n-1}(\omega)$ if and only if $Z_{n-1}(\omega)=z_{n-1}$,

$$
\begin{aligned}
E\left[u\left(Z_{n}\right)-u\left(Z_{n-1}\right)\right]= & E\left[u\left(Z_{n}\right)-u\left(Z_{n-1}\right) \mid Z_{n} \neq Z_{n-1}\right] P\left(Z_{n} \neq Z_{n-1}\right) \\
& +E\left[u\left(Z_{n}\right)-u\left(Z_{n-1}\right) \mid Z_{n}=Z_{n-1}\right] P\left(Z_{n}=Z_{n-1}\right) \\
= & E\left[u\left(Z_{n}\right)-u\left(z_{n-1}\right) \mid Z_{n-1}=z_{n-1}\right] P\left(Z_{n-1}=z_{n-1}\right) .
\end{aligned}
$$

The case $n=M$ is easy: $u$ is affine on the line segment with endpoints $c_{M}$ and $c_{*}$, so $E\left[u\left(Z_{M}\right)-u\left(z_{M-1}\right) \mid Z_{M-1}=z_{M-1}\right]=0$ and

$$
E\left[u\left(Z_{M}\right)-u\left(Z_{M-1}\right)\right]=0 .
$$

Now let $2 \leq n \leq M-1$ and $\gamma_{n}=\frac{z_{n}+z_{n-1}}{2}$. If $n$ is odd, then

$$
\begin{aligned}
E\left[u\left(Z_{n}\right) \mid Z_{n-1}=z_{n-1}\right]= & u\left(z_{n}\right) \frac{x_{n-1}-a_{n}}{x_{n}-a_{n}}+u\left(c_{n}\right) \frac{2 \delta}{x_{n}-a_{n}} \\
= & {\left[u\left(\gamma_{n}\right)+u_{x}\left(\gamma_{n}\right) \delta+\frac{\delta^{2}}{2} R_{n}\right] \frac{x_{n-1}-a_{n}}{x_{n}-a_{n}} } \\
& +\left[u\left(\gamma_{n}\right)-u_{x}\left(\gamma_{n}\right)\left(x_{n-1}+\delta-a_{n}\right)\right] \frac{2 \delta}{x_{n}-a_{n}} \\
= & u\left(\gamma_{n}\right)-u_{x}\left(\gamma_{n}\right) \delta+\frac{\delta^{2}}{2} R_{n} \frac{x_{n-1}-a_{n}}{x_{n}-a_{n}}
\end{aligned}
$$

where, for some $\delta_{n}^{*} \in(0, \delta), R_{n}=u_{x x}\left(\gamma_{n}+\left(\delta_{n}^{*}, 0\right)\right)$. Here

$$
0>R_{n}>u_{x x}\left(\gamma_{n}+(\delta, 0)\right)=-p(p-1)(p-2) \delta x_{n}^{p-3}
$$

and $0>E\left[u\left(Z_{n}\right)-u\left(z_{n-1}\right) \mid Z_{n-1}=z_{n-1}\right]>-C_{p, \theta} \delta^{3}$ follows. But this implies that

$$
\begin{aligned}
E\left[u\left(Z_{n}\right)-u\left(z_{n-1}\right)\right] & >-C_{p, \theta} \delta^{3} P\left(Z_{n-1}=z_{n-1}\right) \\
& >-C_{p, \theta} \delta^{3} .
\end{aligned}
$$

The same inequality is obtained by similar means if $n$ is even. 
Consequently, by (2.11), (2.12), (2.13), (2.2), and $(M-1) \delta=\frac{p-1}{p}-\theta<1$,

$$
\begin{aligned}
\sum_{n=1}^{M} E\left[u\left(Z_{n}\right)-u\left(Z_{n-1}\right)\right] & >-(M-1) C_{p, \theta} \delta^{3} \\
& >-C_{p, \theta}\left(\frac{p}{p-1}-\theta\right)^{3} \frac{1}{(M-1)^{2}} \\
& >-\frac{\theta^{p}}{4}\left(\frac{p}{p-1}\right)^{p-1}
\end{aligned}
$$

and (2.9), hence (2.8), holds. This completes the proof that if $p>2$, then the best constant for the inequality (1.1) is greater than or equal to $p^{p-1} / 2$.

\section{A BiconcAVE MAJORANT OF $v$}

Here $p>2$ and $v$ is the function on $\mathbf{R}^{2}$ defined in the proof of Theorem 1.1. The function identically 1 on $\mathbf{R}^{2}$ is a biconcave majorant of $v$ but more is needed as can be seen from that proof. In this section we show there is a biconcave majorant $u$ of $v$ on $\mathbf{R}^{2}$ with $u(0,0)=0$. Our first step is to prove that there is a function

$$
g:\left[\frac{p-1}{p}, \infty\right) \rightarrow\left[-\frac{p-1}{p}, \infty\right)
$$

with the following properties:

$$
\begin{aligned}
& g \text { is strictly increasing and continuous on }\left[\frac{p-1}{p}, \infty\right), \\
& g(x)>x-2 \text { for all } x \geq \frac{p-1}{p}, \\
& g \text { is differentiable on }\left(\frac{p-1}{p}, \infty\right) \text { and satisfies } \\
& g^{\prime}(x)=\frac{4}{p^{p}(p-1)}(g(x)-x+2)^{-2}(g(x)+1)^{2-p} \text { for all } x>\frac{p-1}{p}, \\
& g\left(\frac{p-1}{p}\right)=-\frac{p-1}{p}, g^{\prime}\left(\frac{p-1}{p}+\right)=\frac{1}{p-1} .
\end{aligned}
$$

An equivalent problem is to show there is a function

$$
G:\left[-\frac{p-1}{p}, \infty\right) \rightarrow\left[\frac{p-1}{p}, \infty\right)
$$

such that

$$
G \text { is strictly increasing and continuous on }\left[-\frac{p-1}{p}, \infty\right) \text {, }
$$

$$
G(y)<y+2 \text { for all } y \geq-\frac{p-1}{p},
$$

$G$ is differentiable on $\left(-\frac{p-1}{p}, \infty\right)$ and satisfies

$$
\begin{aligned}
& G^{\prime}(y)=\frac{p^{p}(p-1)}{4}(y+2-G(y))^{2}(y+1)^{p-2} \text { for all } y>-\frac{p-1}{p}, \\
& G\left(-\frac{p-1}{p}\right)=\frac{p-1}{p}, G^{\prime}\left(-\frac{p-1}{p}+\right)=p-1 .
\end{aligned}
$$

To see the equivalence of the two problems, note that if $g$ satisfies (3.1)-(3.4), then its inverse function satisfies (3.5)-(3.8), and if $G$ satisfies (3.5)-(3.8), then its inverse satisfies (3.1)-(3.4).

Notice that the differential equation for $G$ in (3.7) has the Riccati form. Using the transformation

$$
h(y)=\exp \left[\int_{-\frac{p-1}{p}}^{y} \frac{p^{p}(p-1)}{4}(z+1)^{p-2}(z+2-G(z)) d z\right]
$$


(see, for example, Polyanin and Zaitsev [19]) we obtain the following differential equation for $h(y)$ :

$$
(y+1) h^{\prime \prime}(y)+(2-p) h^{\prime}(y)-\frac{p^{p}(p-1)}{4}(y+1)^{p-1} h(y)=0 .
$$

Two linearly independent solutions of this differential equation on the interval $(-1, \infty)$ are given by

$$
\begin{aligned}
& h_{1}(y)=\sqrt{(1+y)^{p-1}} I_{-\frac{p-1}{p}}\left(z_{0}\right), \\
& h_{2}(y)=\sqrt{(1+y)^{p-1}} I_{\frac{p-1}{p}}\left(z_{0}\right),
\end{aligned}
$$

where $z_{0}=\sqrt{p^{p-2}(p-1)(1+y)^{p}}$ and $I_{\alpha}$ is the modified Bessel function of the first kind (see Abramowitz [1]). Here $\alpha \in\{(p-1) / p,-(p-1) / p\}$,

$$
I_{\alpha}(z)=\sum_{k=0}^{\infty} \frac{\left(\frac{z}{2}\right)^{2 k+\alpha}}{k ! \Gamma(\alpha+k+1)}
$$

and

$$
z^{2} I_{\alpha}^{\prime \prime}(z)+z I_{\alpha}^{\prime}(z)-\left(z^{2}+\alpha^{2}\right) I_{\alpha}(z)=0 .
$$

If $\alpha>-1$, then $I_{\alpha}$ is infinitely differentiable on $(0, \infty)$, which implies that $h_{1}$ and $h_{2}$ are infinitely differentiable on $(-1, \infty)$. By (3.11), $h_{1}$ and $h_{2}$ satisfy (3.10): substitute $h_{i}$ for $h$ on the left side of (3.10) to obtain

$$
\frac{1}{4} p^{2}(1+y)^{\frac{p-3}{2}}\left[z_{0}^{2} I_{\alpha}^{\prime \prime}\left(z_{0}\right)+z_{0} I_{\alpha}^{\prime}\left(z_{0}\right)-\left(z_{0}^{2}+\alpha^{2}\right) I_{\alpha}\left(z_{0}\right)\right]=0 .
$$

Let $h=a_{1} h_{1}+a_{2} h_{2}$ where $a_{1}, a_{2}$ are real numbers such that

$$
h\left(-\frac{p-1}{p}\right)=1 \text { and } h^{\prime}\left(-\frac{p-1}{p}\right)=\frac{p(p-1)}{2} \text {. }
$$

Then, for $y>-1$,

$$
(y+1) h^{\prime \prime}(y)=(p-2) h^{\prime}(y)+\frac{p^{p}(p-1)}{4}(y+1)^{p-1} h(y)
$$

so if we can show that $h>0$ and $h^{\prime}>0$ on $\left[-\frac{p-1}{p}, \infty\right)$, then $h^{\prime \prime}>0$ on $\left[-\frac{p-1}{p}, \infty\right)$ and

$$
h \text { is strictly increasing and convex on }\left[-\frac{p-1}{p}, \infty\right) .
$$

By (3.13) and the continuity of $h^{\prime}$, there is an interval $\left[-\frac{p-1}{p}, c\right)$ on which $h^{\prime}>0$. Let

$$
b=\sup \left\{c: h^{\prime}>0 \text { on }\left[-\frac{p-1}{p}, c\right)\right\} .
$$

Then $h^{\prime}>0$, hence $h>0$, on $\left[-\frac{p-1}{p}, b\right)$, implying, by (3.14), that $h^{\prime \prime}>0$ on $\left[-\frac{p-1}{p}, b\right)$. Therefore, $h^{\prime}$ is strictly increasing on $\left[-\frac{p-1}{p}, b\right)$. Consequently, $b$ is infinite; otherwise, there is a $c>b$ such that $h^{\prime} \geq \frac{p(p-1)}{2}>0$ on $\left[-\frac{p-1}{p}, c\right)$ contradicting the maximality of $b$. So $h, h^{\prime}$, and $h^{\prime \prime}$ are strictly positive on $\left[-\frac{p-1}{p}, \infty\right)$.

These properties of $h$ imply the existence of a function $G$ with the properties (3.5) -(3.8). This function is defined on $\left[-\frac{p-1}{p}, \infty\right)$ by

$$
G(y)=y+2-\frac{4 h^{\prime}(y)}{p^{p}(p-1) h(y)(y+1)^{p-2}} .
$$


The function $g:\left[\frac{p-1}{p}, \infty\right) \rightarrow \mathbf{R}$ is the inverse of $G$. We now define a biconcave majorant $u$ of $v$ on $\mathbf{R}^{2}$, first on the subdomains $D_{0}, \cdots, D_{5}$ :

$$
\begin{aligned}
& D_{1}=\left\{(x, y) \mid 0<x<\frac{p-1}{p},-x<y<\frac{3-p}{p-1} x\right\}, \\
& D_{2}=\left\{(x, y) \mid x>\frac{p-1}{p}, g(x)<y<2 g(x)-x+2\right\}, \\
& D_{3}=\left\{(x, y) \mid x>\frac{p-1}{p}, \frac{1-p}{p} \vee(x-2)<y<g(x)\right\}, \\
& D_{4}=\left\{(x, y) \mid x>\frac{p-1}{p},(-x) \vee(x-2)<y<\frac{1-p}{p}\right\}, \\
& D_{5}=\{(x, y) \mid-x<y<x-2\}, \\
& D_{0}=\{(x, y) \mid x>0,-x<y<x\} \cap\left(\bar{D}_{1} \cup \cdots \cup \bar{D}_{5}\right)^{c} .
\end{aligned}
$$

On $D_{0} \cup \cdots \cup D_{5}$, the function $u$ is defined as follows:

$$
u(x, y)=\left\{\begin{array}{cc}
-\frac{p^{p-1}}{2}\left|\frac{x+y}{2}\right|^{p} & \text { if }(x, y) \in D_{0}, \\
\frac{1}{4}\left(\frac{p}{p-1}\right)^{p-1}[(2-p) x-p y] x^{p-1} & \text { if }(x, y) \in D_{1}, \\
\frac{p^{p-1}}{4}(g(x)+1)^{p-1}((p-2) g(x)-p x+2 p-2) \frac{2(g(x)+1)-x-y}{g(x)+2-x} & \\
-\frac{p^{p-1}}{2}(g(x)+1)^{p} \frac{y-g(x)}{g(x)+2-x} & \text { if }(x, y) \in D_{2}, \\
\frac{p^{p-1}}{4}(y+1)^{p-1}((p-2) y+2 p-2-p G(y)) \frac{y+2-x}{y+2-G(y)} & \\
+\left(1-\frac{p^{p-1}}{2}(y+1)^{p}\right) \frac{x-G(y)}{y+2-G(y)} & \text { if }(x, y) \in D_{3}, \\
\frac{1}{2 p(p-1)}\left(p^{p}(y+1)^{p}-p^{3}(y+1)+2 p(p-1)\right) \frac{y+2-x}{2 y+2} & \text { if }(x, y) \in D_{4}, \\
+\left(1-\frac{p^{p-1}}{2}(y+1)^{p}\right) \frac{x+y}{2 y+2} & \text { if }(x, y) \in D_{5} .
\end{array}\right.
$$

This function $u$ can be extended to a continuous function on $\{(x, y) \mid-x \leq y \leq x\}$. We use the same letter $u$ to denote this extension and define it further on $\mathbf{R}^{2}$ by

$$
u(x, y)=u(y, x)=u(-x,-y)=u(-y,-x) .
$$

The function $u$ is continuous on $\mathbf{R}^{2}$ and the partial derivatives $u_{x}$ and $u_{y}$ exist and are continuous on the set $S=\left\{(x, y) \in \mathbf{R}^{2}:|x-y| \neq 2\right\}$. Furthermore, $u(0,0)=0$. The proof of these properties of $u$ is routine and is omitted. Note that $u$ is not $C^{1}$ on $\mathbf{R}^{2}$. For example, if $y>-\frac{p-1}{p}$, then

$$
\begin{aligned}
u_{x}((y+2)-, y) & =u_{x}((y+2)+, y)+\frac{1}{y+2-G(y)} \\
& >u_{x}((y+2)+, y) .
\end{aligned}
$$

We now show that $u$ is a biconcave majorant of $v$ on $\mathbf{R}^{2}$ and begin by showing that on each of the subdomains $D_{0}, \cdots, D_{5}$ the function $u$ is biconcave. On $D_{0}$ and $D_{5}$, the function $u$ is biconcave since on these subdomains

$$
u_{x x}(x, y)=u_{y y}(x, y)=-\frac{p^{p}(p-1)}{8}\left|\frac{x+y}{2}\right|^{p-2}<0 .
$$

We notice that $u$ is a linear function of $y$ for $(x, y) \in D_{1} \cup D_{2}$, and is a linear function of $x$ for $(x, y) \in D_{3} \cup D_{4}$. So $u_{y y}=0$ on $D_{1} \cup D_{2}$ and $u_{x x}=0$ on $D_{3} \cup D_{4}$. 
Therefore the biconcavity of $u$ on $D_{1}$ and $D_{4}$ follows from the calculations

$$
\begin{aligned}
& u_{x x}(x, y)=-\frac{1}{4}\left(\frac{p}{p-1}\right)^{p-1}\left[p(p-1)(p-2) x^{p-3}\right](x+y)<0 \quad \text { on } \quad D_{1}, \\
& u_{y y}(x, y)=-\frac{1}{4} p^{p}(p-2)(1+y)^{p-3}(x+y)<0 \quad \text { on } \quad D_{4} .
\end{aligned}
$$

Here we have used the assumption that $p>2$. It remains to show that $u_{x x} \leq 0$ on $D_{2}$ and $u_{y y} \leq 0$ on $D_{3}$. The common boundary of $D_{2}$ and $D_{3}$ is the graph of $g$ and the common boundary of $D_{0}$ and $D_{2}$ is the graph of $f$, where $f$ is defined by $f(x)=2 g(x)-x+2($ so $g(x)$ is the average of $f(x)$ and $x-2)$.

We now show that $u_{x x} \leq 0$ on $D_{2}$. If $x \in\left(\frac{p-1}{p}, \infty\right)$ and $g(x)<y<f(x)$, then

$$
\begin{aligned}
u_{x x}(x, y)= & \frac{p^{p}(p-1)}{4}(g(x)+1)^{p-3}\left[-2 g^{\prime}(x)(g(x)+1)\right. \\
& -\left(g^{\prime}(x)\right)^{2}(-2 p+p x+2-2 x-2(p-1) g(x)) \\
& -(-2 g(x)+x-2) g^{\prime \prime}(x)(g(x)+1) \\
& \left.-\left[\left(g^{\prime}(x)\right)^{2}(p-2)+(g(x)+1) g^{\prime \prime}(x)\right] y\right] .
\end{aligned}
$$

This is linear in $y$. Therefore, to show that $u_{x x}(x, y) \leq 0$ for all $(x, y) \in D_{2}$, we need to show only that $u_{x x}(x, f(x)-) \leq 0$ and $u_{x x}(x, g(x)+) \leq 0$. Indeed, $u_{x x}(x, g(x)+)=0$ as can be seen from (3.19) by using the differential equation (3.3) for $g$. Similarly,

$$
u_{x x}(x, f(x)-)=\frac{1}{2} p^{p}(p-1)(g(x)+1)^{p-2}\left(g^{\prime}(x)-1\right) g^{\prime}(x) .
$$

By (3.1) and (3.4), $g(x)+1 \geq \frac{1}{p}>0$ so, by (3.2) and (3.3), $g^{\prime}(x)>0$. Also, as we shall show, $g^{\prime}(x) \leq 1$. Therefore $u_{x x}(x, f(x)-) \leq 0$.

The inequality $g^{\prime}(x) \leq 1$ for all $x \in\left(\frac{p-1}{p}, \infty\right)$ is equivalent to the inequality

$$
G^{\prime}(y) \geq 1 \text { for all } y \in\left(-\frac{p-1}{p}, \infty\right) .
$$

Suppose that (3.20) does not hold. Then there exist numbers $a$ and $b$ such that

$$
\inf \left\{G^{\prime}(y): y>-\frac{p-1}{p}\right\}<b<a<1
$$

Let

$$
\begin{aligned}
& y_{1}=\inf \left\{y>-\frac{p-1}{p}: G^{\prime}(y)=b\right\} \\
& y_{0}=\sup \left\{y>-\frac{p-1}{p}: G^{\prime}(y)=a, y<y_{1}\right\} .
\end{aligned}
$$

Recall that $G^{\prime}\left(-\frac{p-1}{p}\right)=p-1>1$. This and the continuity of $G^{\prime}$ imply that $-\frac{p-1}{p}<y_{0}<y_{1}$. Moreover,

$$
b<G^{\prime}(y)<a=G^{\prime}\left(y_{0}\right)<1
$$

for all $y \in\left(y_{0}, y_{1}\right)$. But there is a number $\varepsilon>0$ such that

$$
\frac{G(y)-G\left(y_{0}\right)}{y-y_{0}}<1 \text { for all } y \in\left(y_{0}, y_{0}+\varepsilon\right)
$$


which, by (3.7), implies that for such $y$,

$$
\begin{aligned}
\frac{4}{p^{p}(p-1)} G^{\prime}(y) & =(y+2-G(y))^{2}(y+1)^{p-2} \\
& =\left(y_{0}+2-G\left(y_{0}\right)+\left(y-y_{0}\right)\left[1-\frac{G(y)-G\left(y_{0}\right)}{y-y_{0}}\right]\right)^{2}(y+1)^{p-2} \\
& >\left(y_{0}+2-G\left(y_{0}\right)\right)^{2}\left(y_{0}+1\right)^{p-2} \\
& =\frac{4}{p^{p}(p-1)} G^{\prime}\left(y_{0}\right),
\end{aligned}
$$

and gives $G^{\prime}(y)>G^{\prime}\left(y_{0}\right)$. For $y \in\left(y_{0}, y_{0}+\varepsilon\right) \cap\left(y_{0}, y_{1}\right)$, this is a contradiction to (3.21). Therefore, (3.20) holds.

Now we check that $u_{y y} \leq 0$ on $D_{3}$. Fix $y \in\left(-\frac{p-1}{p}, \infty\right)$. Then $u_{y y}(G(y)+, y)=0$ and, on $D_{3}, u(x, y)=A(y)+B(y) x$ where $A(y)$ and $B(y)$ are functions of $y$ only. For $G(y)<x<y+2, u_{y y}(x, y)$ is a linear function of $x$. Since $u_{y y}(G(y)+, y)=0$ and

$$
u_{y y}((y+2)-, y)=\frac{2}{(y+2-G(y))^{2}}-\frac{1}{2}(p-1) p^{p}(y+1)^{p-2}=\frac{2\left(1-G^{\prime}(y)\right)}{(y+2-G(y))^{2}},
$$

we see from (3.20) that $u_{y y} \leq 0$ on $D_{3}$.

The function $u$ is biconcave not only on $D_{0}, \cdots, D_{5}$, but also, by (3.17), on each of the reflected subdomains. As can be checked, the function $u$ is concave on each of the horizontal and vertical line segments included in the complement of the union of these open sets. The following elementary lemma easily yields the completion of the proof that $u$ is biconcave on all of $\mathbf{R}^{2}$.

Lemma 3.1. Let $a<c<b$. If $\phi:(a, b) \rightarrow \mathbf{R}$ is concave on $(a, c)$, concave on $(c, b)$, continuous at $c$, differentiable on $(a, c) \cup(c, b)$, and $\phi^{\prime}(c-) \geq \phi^{\prime}(c+)$, then $\phi$ is concave on $(a, b)$.

We now prove that $u$ is a majorant of $v$ on $\mathbf{R}^{2}$. By 3.17), it is enough to prove this for $\{(x, y):-x \leq y \leq x\}$. On $\bar{D}_{0}$ and $\bar{D}_{5}$, we have equality: $u=v$. So by the continuity of $u$ and $v$ on $\left\{(x, y):\left|\frac{x-y}{2}\right|<1\right\}$, it is enough to show in the following that $u \geq v$ on $D_{1}, \cdots, D_{4}$. To do this, we use that $u_{y y}=0$ on $D_{1} \cup D_{2}$ and $u_{x x}=0$ on $D_{3} \cup D_{4}$ as well as $v_{x x}<0$ and $v_{y y}<0$ on $D_{1} \cup \cdots \cup D_{4}$. For example, consider $D_{1}$. On the upper part of its boundary, that is, on $\bar{D}_{0} \cap \bar{D}_{1}$,

$$
u_{y}(x, y)-v_{y}(x, y)=0 .
$$

This follows from the continuity of $u_{y}$ on the closure of $D_{1} \cup D_{2}$ and the equality $u=v$ on $\bar{D}_{0}$. On $D_{1}, u_{y y}(x, y)-v_{y y}(x, y)=-v_{y y}(x, y)>0$, so $u_{y}(x, y)-v_{y}(x, y)$ is strictly increasing in $y$. Therefore, by (3.22),

$$
u_{y}(x, y)-v_{y}(x, y)<0 \text { on } D_{1} .
$$

This implies that $u(x, y)-v(x, y)$ is strictly decreasing in $y$, so the equality $u=v$ on $\bar{D}_{0} \cap \bar{D}_{1}$ gives

$$
u(x, y)-v(x, y)>0 \text { on } D_{1} .
$$

The proof that $u>v$ on $D_{2}$ is the same if $D_{1}$ is replaced by $D_{2}$.

Now consider $D_{3}$. Let $y>-\frac{p-1}{p}$ and let $w$ be the linear function on the interval $[G(y), y+2]$ such that $w(G(y))=u(G(y), y)$ and $w(y+2)=v((y+2)-, y)$. Then, 
as we shall show,

$$
v(x, y)<w(x)<u(x, y)
$$

for all $x \in(G(y), y+2)$, proving that $u>v$ on $D_{3}$. Because $u(\cdot, y)$ is also linear on $[G(y), y+2]$ and

$$
w(y+2)=u(y+2, y)-1<u(y+2, y),
$$

the right side of (3.23) holds. The left side of (13.23) also holds since $v(\cdot, y)$ is strictly concave and

$$
w_{x}((y+2)-)=v_{x}((y+2)-, y) .
$$

This equality can be checked by using

$$
v_{x}((y+2)-, y)=v_{x}((y+2)+, y)=u_{x}((y+2)+, y),
$$

and (3.18), which is equivalent to

$$
\frac{u(y+2, y)-u(G(y), y)}{y+2-G(y)}=v_{x}((y+2)-, y)+\frac{1}{y+2-G(y)} .
$$

By the equality in (3.24), this is equivalent to

$$
\frac{w(y+2)-w(G(y))}{y+2-G(y)}=v_{x}((y+2)-, y) .
$$

Therefore, (3.25) holds and $u>v$ on $D_{3}$.

Now consider the remaining subdomain $D_{4}$. If $y \in\left(-1,-\frac{p-1}{p}\right)$, then $u(\cdot, y)$ is linear and $v(\cdot, y)$ is concave on $[-y, y+2]$. Therefore, $u(\cdot, y)-v(\cdot, y)$ is a convex function on $[-y, y+2]$. To finish the proof that $u>v$ on $D_{4}$, it is enough to observe that

$$
\begin{aligned}
u(-y, y)-v(-y, y) & =\frac{1}{2 p(p-1)}\left(p^{p}(y+1)^{p}-p^{3}(y+1)+2 p(p-1)\right) \\
& \geq \frac{1}{2 p(p-1)}\left[2 p(p-1)-p^{3}(y+1)\right] \\
& \geq \frac{p-2}{2(p-1)}>0
\end{aligned}
$$

and

$$
u_{x}(-y, y)-v_{x}(-y, y)=\frac{p^{2}-p^{p}(y+1)^{p-1}}{4(p-1)}>0 .
$$

This completes the proof that $u$ is a biconcave majorant of $v$ on $\mathbf{R}^{2}$ with $u(0,0)=0$ and the proof of Theorem 1.1.

\section{ConchVity Along the Lines of Positive Slope}

This property of $u$, stronger than biconcavity, will be used in the proof of Theorem 5.1. Here of course $u$ is the function defined in Section 3 and $p>2$.

Lemma 4.1. If $x, y, h, k \in \mathbf{R}$ and $h k \geq 0$, then the function $\varphi: \mathbf{R} \rightarrow \mathbf{R}$ defined by

$$
\varphi(t)=u(x+h t, y+k t)
$$

is concave on $\mathbf{R}$. 
Proof. If $(x, y) \in D_{0} \cup \cdots \cup D_{5}$, then $u_{x x}(x, y) \leq 0, u_{y y}(x, y) \leq 0$, and $u_{x y}(x, y) \leq 0$. The first two inequalities have been proved in Section 3, and the third also holds:

$$
u_{x y}(x, y)= \begin{cases}-\frac{p^{2}}{4}\left(\frac{p}{p-1}\right)^{p-2} x^{p-2} & \text { for }(x, y) \in D_{1}, \\ -\frac{1}{(2-x+g(x))^{2}} & \text { for }(x, y) \in D_{2}, \\ -\frac{1}{(2+y-G(y))^{2}} & \text { for }(x, y) \in D_{3}, \\ -\frac{p^{p}}{4}(1+y)^{p-2} & \text { for }(x, y) \in D_{4}, \\ -\frac{p^{p}(p-1)}{8}\left(\frac{x+y}{2}\right)^{p-2} & \text { for }(x, y) \in D_{0} \cup D_{5} .\end{cases}
$$

Therefore, if $(x+h t, y+k t) \in D_{0} \cup \cdots \cup D_{5}$, then

$$
\varphi^{\prime \prime}(t)=u_{x x}(x+h t, y+k t) h^{2}+2 u_{x y}(x+h t, y+k t) h k+u_{y y}(x+h t, y+k t) k^{2} \leq 0 .
$$

As mentioned in Section $3, u_{x}$ and $u_{y}$ exist and are continuous in the open set $S=\left\{(x, y) \in \mathbf{R}^{2}:|x-y| \neq 2\right\}$. Therefore, the first derivatives of $\varphi$ exist and are continuous on the set $\{t \in \mathbf{R}:(x+h t, y+k t) \in S\}$, so by (4.1) and Lemma 3.1, $\varphi$ is locally concave on this set. It follows from Lemma 3.1 and calculations such as (3.18) that $u$ is concave on the graph $\{(x+h t, y+k t): t \in \mathbf{R}\}$ of any line with $(x, y) \in S$ and $h k \geq 0$. The continuity of $u$ then assures the concavity of $u$ on the graph of each of the lines with $(x, y) \notin S$ and $h=k=1$. Therefore, $\varphi$ is concave on $\mathbf{R}$ under the condition $h k \geq 0$.

In the next section, we shall use the smooth approximation $u^{m}$ of $u$ obtained by convoluting $u$ with the Gaussian density $m \exp \left[-m \pi\left(x^{2}+y^{2}\right)\right]$. The function $u^{m}$ is infinitely differentiable, and $u^{m} \rightarrow u$ pointwise as $m \rightarrow \infty$. Moreover, $u^{m}$ has the concavity property of Lemma 4.1 since the integration that gives $u^{m}$ preserves this property of $u$. Let $h k \geq 0$. Then the function $\varphi_{m}$ associated with $u^{m}$ satisfies $\varphi_{m}^{\prime \prime} \leq 0$ on $\mathbf{R}$, so

$$
u_{x x}^{m}(x, y) h^{2}+2 u_{x y}^{m}(x, y) h k+u_{y y}^{m}(x, y) k^{2} \leq 0 .
$$

Thus, $\varphi_{m}^{\prime}$ is decreasing which gives $\varphi_{m}(1)-\varphi_{m}(0) \leq \varphi_{m}^{\prime}(0)$ by the mean value theorem. Therefore,

$$
u^{m}(x+h, y+k)-u^{m}(x, y) \leq u_{x}^{m}(x, y) h+u_{y}^{m}(x, y) k .
$$

Using the equality $u_{x}^{m}(0,0)=u_{y}^{m}(0,0)=0$, which follows from the analogue of (3.17) for $u^{m}$, and the inequality (4.3), we obtain

$$
u^{m}(h, k) \leq u^{m}(0,0) .
$$

The condition $h k \geq 0$ is necessary for (4.2), (4.3), and (4.4) to hold. Also, there is a positive real number $c_{p}$, the same number for each $m$, such that

$$
\begin{aligned}
\left|u^{m}(x, y)\right| & \leq c_{p}\left(|x|^{p}+|y|^{p}\right)+c_{p}, \\
\left|u_{x}^{m}(x, y)\right| \leq c_{p}\left(|x|^{p-1}+|y|^{p-1}\right)+c_{p}, &
\end{aligned}
$$

with a similar bound for $\left|u_{y}^{m}\right|$. To prove (4.5) and (4.6), use, for example, the biconcavity of $u^{m}$ so that $u_{x}^{m}(\cdot, y)$ is nonincreasing on $\mathbf{R}$, and the definition of $u$ on the set $\left\{(x, y) \in \mathbf{R}^{2}:\left|\frac{x-y}{2}\right|>1\right\}$. 


\section{Differential Subordination}

In this section, we give a simple application of the results of the previous section to martingales $f$ and $g$ where $g$ is not necessarily a transform of $f$. Let $(\Omega, \mathcal{F}, P)$ be a probability space and $\left(\mathcal{F}_{n}\right)_{n \geq 0}$ a filtration of $\mathcal{F}$ : a nondecreasing sequence of sub- $\sigma$-algebras of $\mathcal{F}$. We assume that $f_{n}$ and $g_{n}$ are $\mathcal{F}_{n}$-measurable, $f_{n}=\sum_{k=0}^{n} d_{k}$ and $g_{n}=\sum_{k=0}^{n} e_{k}$ for all nonnegative integers $n$, and that

$$
E\left(d_{n} \mid \mathcal{F}_{n-1}\right)=E\left(e_{n} \mid \mathcal{F}_{n-1}\right)=0
$$

almost everywhere for all $n \geq 1$, that is, $f$ and $g$ are martingales adapted to this filtration. If $f$ and $g$ satisfy

$$
\left|e_{n}(\omega)\right| \leq\left|d_{n}(\omega)\right|
$$

for all $\omega \in \Omega$ and $n \geq 0$, then $g$ is differentially subordinate to $f$. One example is given by $e_{n}=\varepsilon_{n} d_{n}, n \geq 0$, as in Theorem 1.1. Another example, which leads in many contexts to sharp inequalities for stochastic integrals (see, for example, [7], 9], 13]), is given by $e_{n}=v_{n} d_{n}$ where $v_{n}$ is $\mathcal{F}_{(n-1) \vee 0}$-measurable and satisfies $\left|v_{n}(\omega)\right| \leq 1$ for all $\omega \in \Omega$ and all $n \geq 0$.

Theorem 5.1. Let $p>2$ and $\lambda>0$. If $f$ and $g$ are martingales adapted to the same filtration and $g$ is differentially subordinate to $f$, then

$$
\lambda^{p} P\left(g^{*} \geq \lambda\right) \leq \frac{p^{p-1}}{2}\|f\|_{p}^{p}
$$

and the constant $p^{p-1} / 2$ is the best possible.

Proof. That $p^{p-1} / 2$ is a lower estimate for the best constant is a consequence of Theorem 1.1 That it is also an upper estimate can be seen as follows. Assume as before that $\|f\|_{p}$ is finite. As in the proof of Theorem 1.1, it is enough to show that

$$
P\left(\left|g_{n}\right| \geq 1\right) \leq \frac{p^{p-1}}{2}\left\|f_{n}\right\|_{p}^{p} .
$$

This is equivalent to proving that $E v\left(Z_{n}\right) \leq 0$, where $v$ is defined in Section 1, and $Z_{n}$ is defined here by $Z_{n}=\left(X_{n}, Y_{n}\right)$ with $X_{n}=\sum_{k=0}^{n}\left(d_{k}+e_{k}\right)$ and $Y_{n}=$ $\sum_{k=0}^{n}\left(d_{k}-e_{k}\right)$. The function $u$ of Sections 3 and 4 is a majorant of $v$, therefore the theorem will be proved if we can show that

$$
E u\left(Z_{n}\right) \leq 0
$$

for all $n \geq 0$. The first step of the proof of this is to show that

$$
E u^{m}\left(Z_{n}\right) \leq \cdots \leq E u^{m}\left(Z_{0}\right)
$$

for all positive integers $m$ and $n$. Let $H_{n}=d_{n}+e_{n}$ and $K_{n}=d_{n}-e_{n}$. By (5.1), $H_{n} K_{n} \geq 0$, and by (4.3),

$$
\begin{aligned}
u^{m}\left(Z_{n}\right) & =u^{m}\left(X_{n-1}+H_{n}, Y_{n-1}+K_{n}\right) \\
& \leq u^{m}\left(Z_{n-1}\right)+u_{x}^{m}\left(Z_{n-1}\right) H_{n}+u_{y}^{m}\left(Z_{n-1}\right) K_{n} .
\end{aligned}
$$

By (4.5), (4.6), and the finiteness of $\|f\|_{p}$, each term of this inequality has finite expectation. By the martingale condition, $E H_{n}=E K_{n}=0$. Therefore, $E\left[u^{m}\left(Z_{n}\right) \mid \mathcal{F}_{n-1}\right] \leq u^{m}\left(Z_{n-1}\right)$, which implies that $E u^{m}\left(Z_{n}\right) \leq E u^{m}\left(Z_{n-1}\right)$. Consequently, (5.5) holds for $m, n \geq 1$. By (5.1), $X_{0} Y_{0} \geq 0$ which gives, by (4.4), that $E u^{m}\left(Z_{0}\right) \leq u^{m}(0,0)$. Thus, by (5.5),

$$
E u^{m}\left(Z_{n}\right) \leq u^{m}(0,0)
$$


for all $m \geq 1$ and $n \geq 0$. By (4.5),

$$
E\left[\sup _{m \geq 1}\left|u^{m}\left(Z_{n}\right)\right|\right] \leq c_{p}\left(E\left|X_{n}\right|^{p}+E\left|Y_{n}\right|^{p}\right)+c_{p},
$$

in which $E\left|X_{n}\right|^{p}$ and $E\left|Y_{n}\right|^{p}$ are finite, a consequence of the finiteness of $\|f\|_{p}$. Therefore, taking the limit of both sides of (5.6) gives $E u\left(Z_{n}\right) \leq u(0,0) \leq 0$ and (5.4) is proved, which completes the proof of the theorem.

\section{Proof of Theorem 1.2}

The proof has the same pattern as the proof of Theorem 5.1. Let $Z=(X, Y)$ where

$$
X=M+N \quad \text { and } \quad Y=M-N .
$$

The inequality (1.3) will follow if we can show that for $\|M\|_{p}$ finite,

$$
E u^{m}\left(Z_{t}\right) \leq u^{m}(0,0) \text {. }
$$

This holds for $t=0$ just as in Section 5 , so it remains to show that for $t>0$,

$$
E u^{m}\left(Z_{t}\right) \leq E u^{m}\left(Z_{0}\right) .
$$

By Itô's formula as extended by Kunita and Watanabe [18] and Meyer (see [15] and the references given there),

$$
u^{m}\left(Z_{t}\right)=u^{m}\left(Z_{0}\right)+I_{t}+J_{t}+Q_{t} / 2+S_{t},
$$

where

$$
\begin{aligned}
I_{t} & =\int_{(0, t]} u_{x}^{m}\left(Z_{s-}\right) d X_{s}, \\
J_{t} & =\int_{(0, t]} u_{y}^{m}\left(Z_{s-}\right) d Y_{s}, \\
Q_{t} & =\int_{(0, t]}\left(u_{x x}^{m}\left(Z_{s-}\right) d\left[X^{c}, X^{c}\right]_{s}+2 u_{x y}^{m}\left(Z_{s-}\right) d\left[X^{c}, Y^{c}\right]_{s}+u_{y y}^{m}\left(Z_{s-}\right) d\left[Y^{c}, Y^{c}\right]_{s}\right), \\
S_{t} & =\sum_{0<s \leq t}\left(u^{m}\left(Z_{s}\right)-u^{m}\left(Z_{s-}\right)-u_{x}^{m}\left(Z_{s-}\right) \Delta X_{s}-u_{y}^{m}\left(Z_{s-}\right) \Delta Y_{s}\right) .
\end{aligned}
$$

Here $X^{c}$ is the continuous part of $X$ and $\Delta X_{s}=X_{s}-X_{s-}$ for $s>0$. The Bañuelos-Wang condition that $[M, M]_{t}-[N, N]_{t}$ is nonnegative and nondecreasing in $t$ implies that $\left(\Delta M_{s}\right)^{2}-\left(\Delta N_{s}\right)^{2} \geq 0$ for all $s>0$ (see Lemma 1 of Wang [22]). Therefore, $\left(\Delta X_{s}\right)\left(\Delta Y_{s}\right) \geq 0$ and, by (4.3),$S_{t} \leq 0$. Also, $\left[X^{c}, Y^{c}\right]_{s}$, which is equal to $\left[M^{c}, M^{c}\right]_{s}-\left[N^{c}, N^{c}\right]_{s}$, is nonnegative and nondecreasing in $s$. Therefore, $Q_{t} \leq 0$. We now show that $E I_{t}=0$. A similar argument shows that $E J_{t}=0$ so the expectation of the right side of (6.2) is less than or equal to $E u^{m}\left(Z_{0}\right)$ and (6.1) holds. Using the Bañuelos-Wang condition, we see that $[N, N]_{\infty} \leq[M, M]_{\infty}$ so

$$
\begin{aligned}
\|N\|_{2} & =E[N, N]_{\infty} \leq E[M, M]_{\infty} \\
& =\|M\|_{2} \leq\|M\|_{p}<\infty,
\end{aligned}
$$

and $X$, the sum of $M$ and $N$, is a martingale satisfying $\|X\|_{2}<\infty$. Thus, $\left(I_{t}\right)_{t>0}$ is a local martingale (see Theorem 20 on page 56 of [20]). In fact, $\left(I_{t}\right)_{t>0}$ is a martingale as we now show. By [4.6),

$$
\begin{aligned}
\left|u_{x}^{m}\left(Z_{s-}\right)\right| & \leq c_{p}\left(\left|X_{s-}\right|^{p-1}+\left|Y_{s-}\right|^{p-1}\right)+c_{p} \\
& \leq c_{p}\left(\left(X^{*}\right)^{p-1}+\left(Y^{*}\right)^{p-1}\right)+c_{p} .
\end{aligned}
$$


We denote the last expression by $W$. Let $q=p /(p-1)$. By Doob's maximal inequality,

$$
\left\|\left(M^{*}\right)^{p-1}\right\|_{q}^{q}=E\left[\left(M^{*}\right)^{p}\right] \leq q^{p}\|M\|_{p}^{p}<\infty .
$$

Therefore, $\left(M^{*}\right)^{p-1} \in L^{q}$. By a special case of the Burkholder-Davis-Gundy inequality (see, for example, page 287 of [15]),

$$
\begin{aligned}
E\left[\left(N^{*}\right)^{p}\right] & \leq c_{p}^{\prime} E\left([N, N]_{\infty}^{\frac{p}{2}}\right) \\
& \leq c_{p}^{\prime} E\left([M, M]_{\infty}^{\frac{p}{2}}\right) \\
& \leq c_{p}^{\prime \prime} E\left[\left(M^{*}\right)^{p}\right],
\end{aligned}
$$

so $\left(N^{*}\right)^{p-1}$ also belongs to $L^{q}$. This implies that $\left(X^{*}\right)^{p-1}$ and $\left(Y^{*}\right)^{p-1}$ belong to $L^{q}$. Thus, $W \in L^{q}$. Using the Burkholder-Davis-Gundy inequality again, we see that

$$
\begin{aligned}
E\left[\sup _{0<s \leq t}\left|I_{s}\right|\right] & \leq c E\left[\left(\int_{(0, t]} W^{2} d[X, X]_{s}\right)^{\frac{1}{2}}\right] \\
& =c E\left[W\left(\int_{(0, t]} d[X, X]_{s}\right)^{\frac{1}{2}}\right] \\
& \leq c E\left[W\left([X, X]_{t}-[X, X]_{0}\right)^{\frac{1}{2}}\right] .
\end{aligned}
$$

By Hölder's inequality, $E\left[W[X, X]_{\infty}^{\frac{1}{2}}\right] \leq\|W\|_{q}\left\|[X, X]_{\infty}^{\frac{1}{2}}\right\|_{p} \leq c^{\prime}\|W\|_{q}\left\|X^{*}\right\|_{p}<\infty$. Therefore, by the dominated convergence theorem, the right continuity of $[X, X]$, and

$$
\left|E I_{t}\right| \leq c E\left[W\left([X, X]_{t}-[X, X]_{0}\right)\right]
$$

$E I_{t}$ converges to 0 as $t \rightarrow 0$. Moreover,

$$
E\left[\sup _{t>0}\left|I_{t}\right|\right] \leq E\left[W[X, X]_{\infty}^{\frac{1}{2}}\right]<\infty
$$

which implies that the local martingale $\left(I_{t}\right)_{t>0}$ is a martingale. Consequently, $E I_{s}=E I_{t}$ for all $s, t>0$ so $E I_{t}=\lim _{s \rightarrow 0} E I_{s}=0$ completing the proof of (6.1) and Theorem 1.2 .

\section{An INEQUALITY FOR HARMONIC FUNCTIONS}

Let $D$ be an open connected set of points $x=\left(x_{1}, \cdots, x_{n}\right) \in \mathbf{R}^{n}, u$ and $v$ harmonic functions on $D$, and $|\nabla u(x)|$ the Euclidean norm of the gradient vector $\left(u_{x_{1}}(x), \cdots, u_{x_{n}}(x)\right)$. Then $v$ is differentially subordinate to $u$ if, for all $x \in D$,

$$
|\nabla v(x)| \leq|\nabla u(x)| .
$$

Fix a point $\xi \in D$ and let $D_{0}$ be a bounded connected subdomain of $D$ satisfying $\xi \in D_{0} \subset D_{0} \cup \partial D_{0} \subset D$. Denote by $\mu_{D_{0}}^{\xi}$ the harmonic measure on $\partial D_{0}$ with respect to $\xi$. If $1 \leq p<\infty$, let

$$
\|u\|_{p}=\sup _{D_{0}}\left[\int_{\partial D_{0}}|u(x)|^{p} d \mu_{D_{0}}^{\xi}(x)\right]^{\frac{1}{p}}
$$

where the supremum is taken over all such $D_{0}$. Assume that $|v(\xi)| \leq|u(\xi)|$.

For any such $D_{0}$, let $\tau(\omega)=\inf \left\{t \geq 0:\left|Z_{t}\right| \notin D_{0}\right\}, M_{t}=u\left(Z_{t \wedge \tau}\right)$, and $N_{t}=v\left(Z_{t \wedge \tau}\right)$, where $\left(Z_{t}\right)_{t \geq 0}$ is a Brownian motion in $\mathbf{R}^{n}$ that starts at $\xi: Z_{0}=\xi$. 
Then $M$ and $N$ are martingales. They also satisfy the Bañuelos-Wang condition as can be seen from

$$
\begin{aligned}
{[M, M]_{t} } & =|u(\xi)|^{2}+\int_{0}^{t \wedge \tau}\left|\nabla u\left(Z_{s}\right)\right|^{2} d s \\
{[N, N]_{t} } & =|v(\xi)|^{2}+\int_{0}^{t \wedge \tau}\left|\nabla v\left(Z_{s}\right)\right|^{2} d s .
\end{aligned}
$$

Theorem 7.1. Let $p>2$. If $u$ and $v$ are harmonic functions on $D$ such that $|v(\xi)| \leq|u(\xi)|$ for some $\xi \in D_{0}$ as above, and $v$ is differentially subordinate to $u$, then

$$
\mu_{D_{0}}^{\xi}\left(\left\{x \in \partial D_{0}:|v(x)| \geq 1\right\}\right) \leq \frac{p^{p-1}}{2}\|u\|_{p}^{p}
$$

Proof. Since $D_{0}$ is bounded, we have that $P(\tau<\infty)=1, P\left(Z_{\tau} \in \partial D_{0}\right)=1, M$ is a uniformly integrable martingale, and $\|M\|_{p}=\left\|u\left(Z_{\tau}\right)\right\|_{p}<\infty$. The distribution of $Z_{\tau}$ on $\partial D_{0}$ is the harmonic measure $\mu_{D_{0}}^{\xi}$. Therefore, by Theorem 1.2,

$$
\begin{aligned}
\mu_{D_{0}}^{\xi}\left(\left\{x \in \partial D_{0}:|v(x)| \geq 1\right\}\right) & =P\left(\left|v\left(Z_{\tau}\right)\right| \geq 1\right) \\
& \leq P\left(N^{*} \geq 1\right) \\
& \leq \frac{p^{p-1}}{2}\|M\|_{p}^{p} \\
& =\frac{p^{p-1}}{2} \int_{\partial D_{0}}|u(x)|^{p} d \mu_{D_{0}}^{\xi}(x) \\
& \leq \frac{p^{p-1}}{2}\|u\|_{p}^{p} .
\end{aligned}
$$

Remark. The best constant for the inequality (7.2) is unknown if $n>2$ and $p>2$. It is also unknown if $n>2$ and $1<p<2$ (but see [21). If $n \geq 1$ and $p=1$, it is known to be 2 (see [10] and page 1023 of [12]). In the classical case in which $n=2$, $D$ is the open unit disk, $u$ is harmonic on $D, v$ is its conjugate with $v(0)=0$, and $\xi=0$, Davis proved that for $p=1$, the best constant is

$$
\frac{1+\frac{1}{3^{2}}+\frac{1}{5^{2}}+\frac{1}{7^{2}}+\cdots}{1-\frac{1}{3^{2}}+\frac{1}{5^{2}}-\frac{1}{7^{2}}+\cdots} .
$$

Later Baernstein 2] gave another proof. Modifying Baernstein's method, Tomaszewski [21] found the best constant in a related case in which $1<p<2$. In the conjugate harmonic function case, the best constant for $p>2$ is still unknown as far as we know, although Essén [17] has some deep results that may lead to the answer.

\section{ACKNOWLEDGEMENT}

The author is pleased to acknowledge her indebtedness to Professor Donald L. Burkholder for his support and encouragement during the course of this work, which is a part of the author's doctoral dissertation. 


\section{REFERENCES}

[1] M. Abramowitz and I. A. Stegun, editors, Handbook of Mathematical Functions with formulas, graphs, and mathematical tables, Reprint of the 1972 edition, Dover Publications, Inc., New York, 1992. MR94b:00012

[2] A. Baernstein, Some sharp inequalities for conjugate functions, Indiana Univ. Math. J. 27 (1978), no. 5, 833-852. MR80g:30022

[3] R. Bañuelos and G. Wang, Sharp inequalities for martingales with applications to the Beurling-Ahlfors and Riesz transforms, Duke Math. J. 80 (1995), no 3, 575-600. MR $96 \mathrm{k}: 60108$

[4] R. Bañuelos and G. Wang, Orthogonal martingales under differentiable subordination and applications to Riesz transforms, Illinois J. Math. 40 (1996), 678-691. MR99a:60047

[5] R. Bañuelos and G. Wang, Davis's inequality for orthogonal martingales under differential subordination, Michigan Math. J. 47 (2000), 109-124. MR2001g:60100

[6] D. L. Burkholder, Martingale transforms, Ann. Math. Statist. 37 (1966), 1494-1504. MR 34:8456

[7] D. L. Burkholder, Boundary value problems and sharp inequalities for martingale transforms, Ann. Probab. 12 (1984), 647-702. MR:86b:60080

[8] D. L. Burkholder, A sharp and strict $L^{p}$-inequality for stochastic integrals, Ann. Probab. 15 (1987), 268-273. MR88d:60156

[9] D. L. Burkholder, Sharp inequalities for martingales and stochastic integrals, Astérisque 157-158 (1988), 75-94. MR90b:60051

[10] D. L. Burkholder, Differential subordination of harmonic functions and martingales, Harmonic Analysis and Partial Differential Equations (El Escorial, 1987), Lecture Notes in Math. 1384 (1989), 1-23. MF90k:31004

[11] D. L. Burkholder, Explorations in martingale theory and its applications, Lecture Notes in Math. 1464 (1991), 1-66. MF.92m:60037

[12] D. L. Burkholder, Strong differential subordination and stochastic integration, Ann. Probab. 22 (1994), 995-1025. MR.95h:60085

[13] D. L. Burkholder, Sharp norm comparison of martingale maximal functions and stochastic integrals, Proceedings of the Norbert Wiener Centenary Congress (East Lansing, MI, 1994), 343-358, Proc. Sympos. Appl. Math. 52, Amer. Math. Soc., Providence, RI (1997). MR 98f:60103

[14] B. Davis, On the weak type $(1,1)$ inequality for conjugate functions, Proc. Amer. Math. Soc. 44 (1974), 307-311. MR 50:879

[15] C. Dellacherie and P. A. Meyer, Probability and Potential: The Theory of Martingales, North-Holland, Amsterdam, 1982. MR85e:60001

[16] J. L. Doob, Stochastic Processes, Wiley, New York, 1953. MR 15:445b

[17] M. Essén, On sharp constants in the weak type $(p, p)$-inequalities, $2<p<\infty$, Report No. 43 1999/2000, Institut Mittag-Leffler.

[18] H. Kunita and S. Watanabe, On square integrable martingales, Nagoya Math. J. 30 (1967), 209-245. MR 36:945

[19] A. D. Polyanin and V. F. Zaitsev, Handbook of Exact Solutions for Ordinary Differential Equations, CRC Press, 1995. MR98c:34001

[20] P. Protter, Stochastic Integration and Differential Equations: A New Approach, SpringerVerlag, Berlin, 1990. MR.91i:60148

[21] B. Tomaszewski, Some sharp weak-type inequalities for holomorphic functions on the unit ball of $\mathbf{C}^{n}$, Proc. Amer. Math. Soc. 95 (1985), 271-274. MR87a:32005

[22] G. Wang, Differential subordination and strong differential subordination for continuous-time martingales and related sharp inequalities, Ann. Probab. 23 (1995), 522-551. MR96b:60120

Department of Statistics, Purdue University, West Lafayette, Indiana 47907

E-mail address: jsuh@stat.purdue.edu 